\title{
Comparison of Scintillators for Positron Emission Mammography (PEM) Systems
}

\author{
Raymond R. Raylman, Member, IEEE, Stan Majewski, Member, IEEE, Mark F. Smith, Member, IEEE, Randy Wojcik, \\ Andrew G. Weisenberger, Member, IEEE, Brian Kross, Vladimir Popov, and Jamal J. Derakhshan
}

\begin{abstract}
Positron emission mammography (PEM) has promise as an effective method for the detection of breast lesions. Perhaps the most significant design feature of a PEM system is the choice of scintillator material. In this investigation we compared three scintillators for use in PEM: NaI(TI), gadolinium oxyorthosilicate (GSO), and lutetium-gadolinium oxyorthosilicate (LGSO). The PEM systems consisted of two $30 \times 30$ arrays of pixelated scintillators $\left(3 \times 3 \times 10 \mathrm{~mm}^{3}\right.$ for GSO and LGSO and $3 \times 3 \times 19 \mathrm{~mm}^{3}$ for $\mathrm{NaI}(\mathrm{TI})$ ) coupled to arrays of square position-sensitive photomultiplier tubes. The Compton scatter fraction, system energy resolution, spatial resolution, spatial resolution uniformity, and detection sensitivity were compared. Compton scatter fractions for the systems were comparable, between $8 \%$ and $9 \%$. The $\mathrm{NaI}(\mathrm{Tl})$ system produced the best system energy resolution $(\mathbf{1 8 . 2 \%})$, the GSO system had the worst system energy resolution $(\mathbf{2 8 . 7 \%})$ ). Spatial resolution for each system was relatively uniform across the face of the detectors, though the magnitude was dependent upon scintillator material. The NaI(TI) system produced the lowest mean resolution $(3.54 \pm 0.05 \mathrm{~mm}$ for horizontal profiles and $3.51 \pm \mathbf{0 . 0 4} \mathbf{~ m m}$ for vertical profiles), while the LGSO system produced the greatest mean spatial resolution $(3.19 \pm 0.04 \mathrm{~mm}$ for horizontal profiles and $3.20 \pm 0.03 \mathrm{~mm}$ for vertical profiles). Detection sensitivity varied among the three systems: $\mathrm{NaI}(\mathrm{Tl})=217.7 \mathrm{c} / \mathrm{s} / \mathrm{kBq} / \mathrm{ml}, \mathrm{GSO}=383.9 \mathrm{c} / \mathrm{s} / \mathrm{kBq} / \mathrm{ml}$ and LGSO $=646.9 \mathrm{c} / \mathrm{s} / \mathrm{kBq} / \mathrm{ml}$. Imaging of a simulated breast containing various sized spheres demonstrated that the LGSO system produced the greatest detectability for small spheres (as gauged by the contrast-to-noise ratio), while the NaI(Tl) system had the worst detectability. These differences were due mainly to the lower sensitivity of the NaI(TI) system compared to the LGSO and GSO imagers. This investigation demonstrated the very important connection between scintillator selection and performance of PEM systems.
\end{abstract}

Index Terms-Breast imaging, nuclear medicine, scintillators.

\section{INTRODUCTION}

$\mathbf{T}$ HE effective application of ${ }^{18} \mathrm{~F}$-Fluorodeoxyglucose (FDG)-positron emission tomography (PET) imaging to the detection of breast cancer has motivated the development

Manuscript received June 5, 2002; revised September 21, 2002. This work was supported in part by the National Cancer Institute (R21 CA82752-01), the Southeastern Universities Research Association (SURA) operates the Thomas Jefferson National Accelerator Facility for the United States Department of Energy under contract DE-AC05-84ER40150, and by the Office of Biological and Environmental Research of the Office of Science of the U.S. Department of Energy.

R. R. Raylman and J. J. Derakhshan are with the Center for Advanced Imaging, Department of Radiology, Robert C. Byrd Health Sciences Center, West Virginia University, Morgantown, WV 26506 USA (e-mail: rraylman@wvu.edu)

S. Majewski, M. F. Smith, R. Wojcik, A. G. Weisenberger, B. Kross, and V. Popov are with the Thomas Jefferson National Accelerator Facility, Newport News, VA 23606 USA.

Digital Object Identifier 10.1109/TNS.2002.807943 of specialized breast imaging methods. Perhaps the most promising of these new techniques is positron emission mammography (PEM). While most of the proposed PEM systems have virtually the same geometry (two opposing arrays of scintillator coupled to position-sensitive photomultiplier tubes), the choice of scintillator has varied. For example, Thompson, et al. used blocks of the PET scintillator Bismuth Germanate (BGO) scored on the top and bottom surfaces to create offset arrays of $2 \times 2 \times 20 \mathrm{~mm}^{3}$ pixels [1], [2]. Groups from UCLA and Berkeley have proposed the use of Cerium-doped Lutetium Oxyorthosilicate (LSO) [3], [4]. The potential advantage of LSO compared to BGO is its much higher light output (approximately a factor of five greater) and shorter primary light pulse decay constant (40 ns versus $300 \mathrm{~ns}$ ) [5]. A potentially negative quality of LSO is the presence of ${ }^{176} \mathrm{Lu}$, which emits electrons (average emission energies of $50 \mathrm{keV}$ and $181 \mathrm{keV}$ ) and a number of gamma rays (energies range from 88 to $401 \mathrm{keV}$ ). Another promising Cerium-doped Lutetium-based orthosilicate is Lutetium-Gadolinium Oxyorthosilicate (LGSO) [6]. LGSOs scintillation properties are slightly less favorable than those of LSO. Specifically, the scintillation light output from LGSO is $20 \%$ lower than LSO. In addition, the photofraction and attenuation coefficients of LGSO are lightly lower than LSO. LGSO, however, contains approximately $10 \%$ less ${ }^{176} \mathrm{Lu}$ than LSO, thus the potential for increased deadtime and random coincidence rates caused by the intrinsic radioactivity are reduced. Gadolinium Oxyorthosilicate (GSO) has also been used in PEM imagers [7]. This scintillator does not contain ${ }^{176} \mathrm{Lu}$, so it is not intrinsically radioactive. GSO produces less light than LSO (approximately 2.5 times less light) and has a slightly longer decay constant than LSO (60 ns versus $40 \mathrm{~ns}$ ). $\mathrm{NaI}(\mathrm{Tl})$ has also been proposed for use in PEM imagers [8]. Thallium-activated Sodium Iodide has been a staple scintillator in gamma cameras for many years. Its use offers extremely high light output (33\% greater than LSO) and lower cost. Unfortunately, $\mathrm{NaI}(\mathrm{Tl})$ possesses low stopping power for annihilation photons (approximately 60\% less than LSO) and very long light pulse decay constants (230 ns primary and $\sim 10,000 \mathrm{~ns}$ secondary).

In this investigation, we compared the performance of three representative scintillators ( $\mathrm{NaI}(\mathrm{Tl}), \mathrm{GSO}$, and LGSO) applied to PEM imaging. Specifically, spatial resolution, detection sensitivity, scatter fraction, and system energy resolution were measured for each scintillator. Finally, the imaging performance of the scintillators was compared by scanning a realistic breast phantom containing simulated breast tumors. 
TABLE I

CHARACTERISTICS FOR DETECTION OF 511 KEV PHOTONS FOR THE THREE SCINTILLATORS TESTED

\begin{tabular}{|c|c|c|c|}
\hline & $\mathrm{NaI}(\mathrm{Tl})$ & GSO & LGSO* \\
\hline Density $\left(\mathrm{g} / \mathrm{cm}^{3}\right)$ & 3.67 & 6.71 & 7.40 \\
\hline Effective Atomic Number & 51 & 59 & 66 \\
\hline \multicolumn{4}{|l|}{ Total Linear Attenuation } \\
\hline Coefficient $[\mu]\left(\mathrm{cm}^{-1}\right)$ & 0.331 & 0.684 & 0.821 \\
\hline \multicolumn{4}{|l|}{ Product of $\mu$ and Pixel } \\
\hline Thickness & 0.629 & 0.684 & 0.821 \\
\hline Photofraction (\%) & 18.2 & 27.0 & 34.8 \\
\hline
\end{tabular}

\section{MethodS}

\section{A. Scintillator Arrays}

For each scintillator type (Cerium-doped Gadolinium Oxyorthosilicate (GSO), Cerium-doped Lutetium-Gadolinium Oxyorthosilicate (LGSO) and Thallium-activated Sodium Iodide $(\mathrm{NaI}(\mathrm{Tl}))$, two $30 \times 30$ arrays of discrete scintillator pixels were constructed. Some relevant parameters for these scintillators are listed in Table I. The cross section of each pixel was $3 \times 3 \mathrm{~mm}^{2}$. This pixel size is representative of the pixel cross sections currently used or proposed for use in PEM imagers [2]-[4], [7], [8]. Pixel thickness was $10 \mathrm{~mm}$ for GSO and LGSO and $19 \mathrm{~mm}$ for $\mathrm{NaI}(\mathrm{Tl})$. The thicker $\mathrm{NaI}(\mathrm{Tl})$ was intended to offset the significantly lower attenuation coefficient of NaI(Tl) compared to GSO and LGSO (Table I). The sides of the crystals in contact with the light collection units (described below) were polished; all other surfaces had a rough-cut finish. For the GSO and LGSO arrays, each crystal was wrapped with white Teflon tape $(0.15 \mathrm{~mm}$ thick) to optically separate individual pixels and to improve light collection through diffusive reflection. The crystal arrays were formed using a frame constructed from thin nylon rods. For the $\mathrm{NaI}(\mathrm{Tl})$ array, the crystals were mounted in a $30 \times 30$ grid of Teflon and sealed to prevent absorption of water by the highly hygroscopic scintillator. Due to the thickness of the Teflon, the average pixel spacing for all of the detector arrays was $3.3 \mathrm{~mm}$. The scintillator arrays were coupled to the light collectors through $1 \mathrm{~cm}$ thick acrylic light diffusers. These diffusers dispersed the light from the detectors so that a number of photomultiplier elements received scintillation light. This scheme helped eliminate loss of signal from scintillators located at seams in the PSPMT arrays.

\section{B. Light Collection}

The scintillator arrays were optically coupled through the diffusers to light collection devices to create the detector heads. Each of these units consisted of a $4 \times 4$ array of compact Hamamatsu R5900-C8 position-sensitive photomultipliers (PSPMT). The flangeless version of this photomultiplier, with a $22 \mathrm{~mm}$ square photocathode size, permitted tight arrangement of the PSPMT array with a step of $26.1 \mathrm{~mm}$. Readout of the arrays (each PSPMT having $8(4(X)+4(Y))$ output anode wires) was

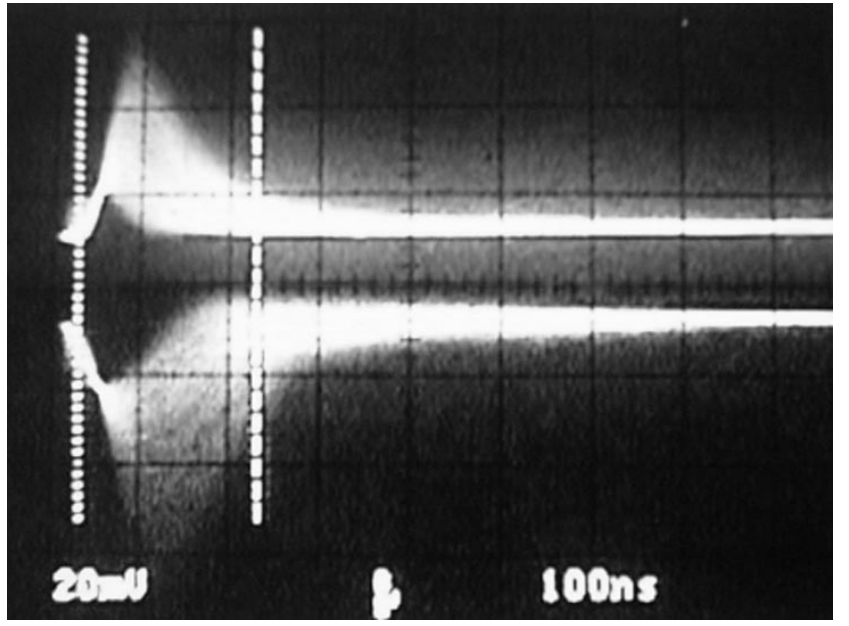

Fig. 1. Output pulses for the GSO system (top curves) and NaI(Tl) system (bottom curves).

simplified by interconnecting corresponding $X$ and $Y$ wires from different PSPMTs to form $16(X) \times 16(Y)$ combined anode outputs. Each combined $X$ and $Y$ anode output was amplified by a custom amplifier board located in the detector head. The $10 \mathrm{~ns}$ pulses produced by each detector head were sent to a coincidence unit producing $100 \mathrm{~ns}$ trigger pulses on detection of coincidences. To estimate the random coincidence rate, the signals from one detector head were split off, delayed and placed in coincidence with the undelayed signals from the other head. Thus, any resulting coincidence must be accidental. The total energy of each event was measured and an energy threshold window applied. The energy windows were centered on the photopeaks of the spectra from each detector $(400-650 \mathrm{keV}$ for $\mathrm{NaI}(\mathrm{Tl}), 350-700 \mathrm{keV}$ for GSO and LGSO). The energy data were histogrammed to produce a spectrum for each detector head. Each imager had a square field-of-view (FOV) measuring $10 \times 10 \mathrm{~cm}^{2}$. Each set of PEM detector heads was mounted on a special fixture and separated by $20 \mathrm{~cm}$ to simulate use in a PEM biopsy apparatus. Since the detector size was $10 \times 10 \mathrm{~cm}^{2}$ and the separation was $20 \mathrm{~cm}$, the maximum possible coincidence angle for true events was $\tan ^{-1}\left(\sqrt{ }\left(2^{*} 10^{2}\right) / 20\right)=35.3^{\circ}$. Due to the slow decay of the light pulses emitted by $\mathrm{NaI}(\mathrm{Tl})$, pulse clipping and shaping circuitry [dual-stage shaping amplifiers (320 ns shaping time)] were utilized. As shown in Fig. 1, this technique was effective in reducing the width of the output pulses produced by the $\mathrm{NaI}(\mathrm{Tl})$-based PEM system. The output pulses from the GSO, LGSO and $\mathrm{NaI}(\mathrm{Tl})$ detectors were of equivalent length. Since the pulse shapes of all of the scintillators were approximately the same, a constant pulse integration time (175 ns) was utilized in all three systems. This integration time was selected as a compromise between the need for the collection of all charge and the desire to reduce pulse pile-up.

\section{Data Acquisition}

The data acquisition system was based on the versa module Eurocard (VME) data bus. This bus architecture is capable of performing extremely high data transfer and processing rates. A Motorola MVME2700-1431 VME 366MHz G3 CPU controlled data acquisition. The CPU used the VxWorks real-time 
operating system from Wind Rivers Systems, Inc. In addition, the VME CPU provided processed image data to a Macintosh G4 workstation. Two CAEN, Inc. V785 32 channel analog-todigital converters (ADCs) were used to read out the analog channels of the detector readout system. Control of data acquisition was performed with software developed using the new Kmax 7.0 development package from Sparrow, Inc. installed on the G4 Macintosh. The G4 computer was interfaced to the VME CPU via high speed Ethernet. The new cross platform version of Kmax implements a client/server architecture that permits a user operating on one computer to connect via Ethernet to hardware attached to another computer, in this case, the VME CPU. Data were acquired in list mode to allow reconstruction with an iterative algorithm.

\section{Image Reconstruction}

Image reconstruction was performed using the maximum likelihood expectation maximization (MLEM) algorithm [9] optimized for use with these PEM systems. The matrix equation $y=G s$ was solved for activity in a three-dimensional source region between the two detectors. In this relationship $y$ is a list of the coincidences along each line of response, $s$ is a vector representing activity in voxels in the discretized source region and $G$ is the system matrix. The system matrix elements were computed using ray-tracing methods between detector pixels. All lines of response were used in the reconstruction. A total of six iterations were performed. No Compton scatter or photon attenuation corrections were applied. Events from the random event channel were also reconstructed with the MLEM algorithm and subtracted from the prompt coincidence image. Images were reconstructed into a $60 \times 60 \times 60 \times$ pixel matrix (each pixel was $1.66 \times 1.66 \times 1.66 \mathrm{~mm}^{3}$ ). The reconstructed images were displayed and analyzed utilizing software created with the Interactive Data Language (IDL) programming environment implemented on a Macintosh G4 computer.

\section{E. Scintillator Evaluation}

1) Resolution: The procedures for measuring resolution and resolution uniformity were based on techniques described in the National Electronics Manufacturers Association (NEMA) publication NU2-1994 [10]. Point sources were made by soaking ceramic beads (diameter $=1.5 \mathrm{~mm}$ ) in a solution containing ${ }^{18} \mathrm{~F}$. A cross pattern was created by attaching seventeen beads to a thin copper-clad board. Eight beads were placed along the vertical ( $y$-axis) and eight along the horizontal ( $x$-axis), with one in the center of the field of view (FOV); bead spacing was $1 \mathrm{~cm}$. Thus, it was possible to measure the mean spatial resolution at radial positions of $1,2,3$, and $4 \mathrm{~cm}$ from the center of the FOV. At least 850000 counts were acquired so that there were approximately 50000 counts in the image of each source. The data were reconstructed to produce images of the cross pattern. Horizontal and vertical profiles were drawn on the images of the point sources to produce point spread functions (PSFs). Thus, for each radial distance there were four measurements for each of the two profiles, except the center source that had one profile per profile direction.

2) Compton Scatter Fraction: The Compton scatter fraction was measured using a three-compartment phantom $\left(9 \times 14 \times 5 \quad \mathrm{~cm}^{3}\right) \quad$ [11]. Each compartment measured $3 \times 14 \times 5 \mathrm{~cm}^{3}$. The central compartment was filled with water containing no radioactivity. The two compartments to either side of the central compartment were filled with water containing $1.5 \mathrm{MBq}$ of FDG. The phantom was placed at the center of the imager and a minimum of 500000 counts acquired. The data were reconstructed using the method previously described. The scatter fraction was calculated by dividing the total number of counts present in the image of the central "cold" compartment by the average of the total counts present in the two "hot" compartments.

3) System Energy Resolution: The energy resolution of the PEM system with each of the three scintillators was measured by imaging a uniform flood phantom $\left(10 \times 10 \times 6 \mathrm{~cm}^{3}\right)$ filled with $370 \mathrm{kBq}(10 \mu \mathrm{Ci})$ of ${ }^{18} \mathrm{~F}$. The phantom was imaged until a total of at least 50000 counts were acquired in each of the energy spectra. The photopeaks of the spectra were fit with Gaussian functions. The system energy resolution was calculated using

$$
E_{\mathrm{res}}(\%)=\frac{\mathrm{FWHM}}{511} * 100 .
$$

FWHM is the full width at half maximum of the photopeak.

4) Detection Sensitivity: The detection sensitivity of the PEM systems with each of the three scintillators was measured using a procedure based on the NEMA protocol for measurement of PET scanner sensitivity [10]. The most significant deviation from the NEMA protocol was the utilization of a rectangular flood phantom $\left(10 \times 10 \times 6 \mathrm{~cm}^{3}\right)$ instead of a cylindrical phantom. The rationale for this substitution is the fact that PEM utilizes stationary planar detectors, thus a planar source is more appropriate for detection sensitivity measurements than is a cylinder. In addition, since breast compression is usually applied in PEM imaging protocols, the planar source more closely resembles the imaging conditions encountered by PEM in clinical use. Approximately $370 \mathrm{kBq}(10 \mu \mathrm{Ci})$ of ${ }^{18} \mathrm{~F}$ were placed in the flood phantom. The phantom was positioned at the center of the imager and a 300s data acquisition performed. The data were not reconstructed. Instead, the total number of detected true counts was calculated by subtracting the number of delayed channel events from the prompt events. At the radioactivity level present in the phantom, system deadtime was less than $1 \%$. Detection sensitivity was calculated using the equation [10]

$$
S=\frac{C_{\text {true }}}{T_{\text {acq }}}\left(\frac{1-S F}{a_{\text {ave }}}\right)
$$

where $C_{\text {true }}$ is the number of true and random events, $T_{\text {acq }}$ is the acquisition duration (300 s), SF is the scatter fraction and $a_{\text {ave }}$ is the average concentration of ${ }^{18} \mathrm{~F}$ present in the phantom during the data acquisition given by [10]

$$
a_{\text {ave }}=\frac{A_{o}}{\ln 2^{*} V}\left(\frac{T_{1 / 2}}{T_{\text {acq }}}\right)\left\{1-\exp \left(\frac{-T_{\text {acq }}}{T_{1 / 2}} \ln 2\right)\right\}
$$

where $A_{O}$ is the amount of activity at the start of the data acquisition, $V$ is the volume of the flood phantom and $T_{1 / 2}$ is the half-life of ${ }^{18} F$ (6600 s).

5) Lesion Detectability: Ultimately the effectiveness of a PEM system must be assessed by its ability to successfully detect radiotracer-avid breast lesions. In order to evaluate 


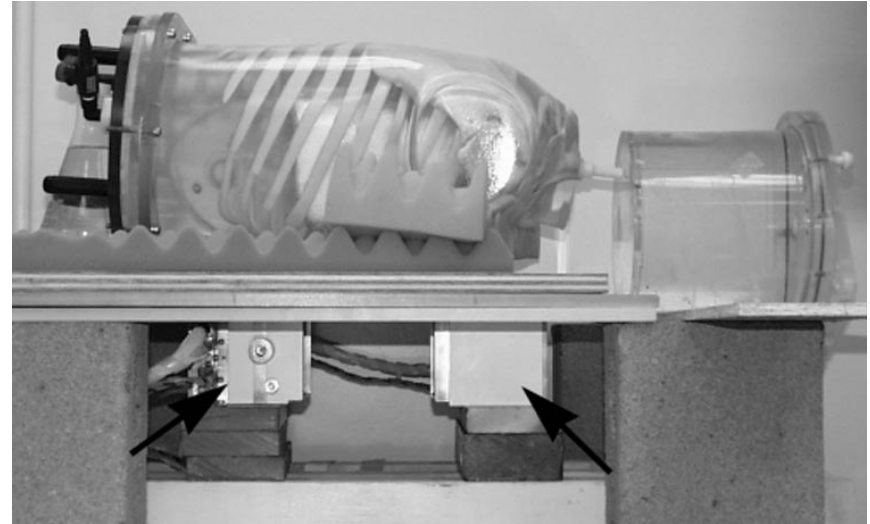

Fig. 2. Picture showing the PEM detectors and the phantoms simulating background uptake of FDG. The arrows show the positions of the detector heads.

this capability, a phantom simulating a compressed breast containing lesions was imaged. Specifically, four spheres with diameters of $5,9,12$, and $15 \mathrm{~mm}$ were filled with a solution containing ${ }^{18} \mathrm{~F}$ (concentration representative of that reported for breast cancers $(20.35 \mathrm{kBq} / \mathrm{ml} 0.55 \mu \mathrm{Ci} / \mathrm{ml})$ [12]) and imbedded in a $5 \mathrm{~cm}$ thick block of gelatin (simulating a compressed breast). The concentration of ${ }^{18} \mathrm{~F}$ in the gelatin block $(2.39 \mathrm{kBq} / \mathrm{ml}[0.065 \mu \mathrm{Ci} / \mathrm{ml}])$ was representative of that reported in human studies one hour after injection of $370 \mathrm{MBq}$ of FDG [13]. The target-to-background concentration ratio was $8.5: 1$. The simulated breast phantom was placed at the center of the imager and data acquired for $300 \mathrm{~s}$. Accumulation of FDG in the normal organs of the body was simulated with an anthropomorphic torso phantom. The tank of the torso phantom (simulating adipose and muscle tissue in the upper torso) contained $55.5 \mathrm{MBq}$ of ${ }^{18} \mathrm{~F}$, the simulated liver $18.5 \mathrm{MBq}$ and the simulated myocardium with $11.3 \mathrm{MBq}$. These amounts represent the upper limits of tracer uptake measured one hour after injection of $370 \mathrm{MBq}$ of FDG [14], [15]. Accumulation of FDG in the brain and bladder were simulated with a $20 \mathrm{~cm}$ diameter cylindrical flood phantom and a $500 \mathrm{ml}$ beaker, respectively. The torso phantom, flood phantom and beaker were placed on a platform located above the imager (Fig. 2). Images were reconstructed using the MLEM algorithm previously described. Regions of interest (ROIs) were drawn on the image of each sphere and on a background region adjacent the spheres. A task-dependent metric [contrast-to-noise ratio (CNR)] was used to quantify lesion detectable. This quantity is given by

$$
C N R=\frac{S_{\mathrm{sp}}-S_{\mathrm{bkg}}}{\sigma_{\mathrm{bkg}}}
$$

where $S_{\mathrm{sp}}$ is the mean pixel value in a region-of-interest (ROI) drawn on the image of a sphere, $S_{\mathrm{bkg}}$ is the mean pixel value from an ROI drawn in a background region and $\sigma_{\mathrm{bkg}}$ is the standard deviation in the pixel values calculated for the ROI placed in the background region.

\section{RESULTS}

Fig. 3 shows the cross-pattern point source phantom used to measure resolution and resolution uniformity [Fig. 3(a)]. Also

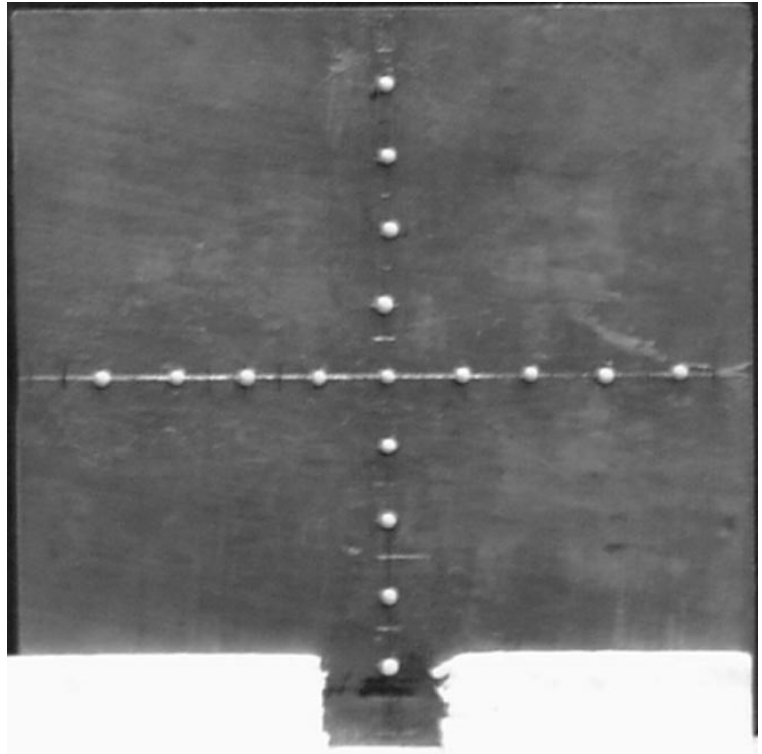

(a)

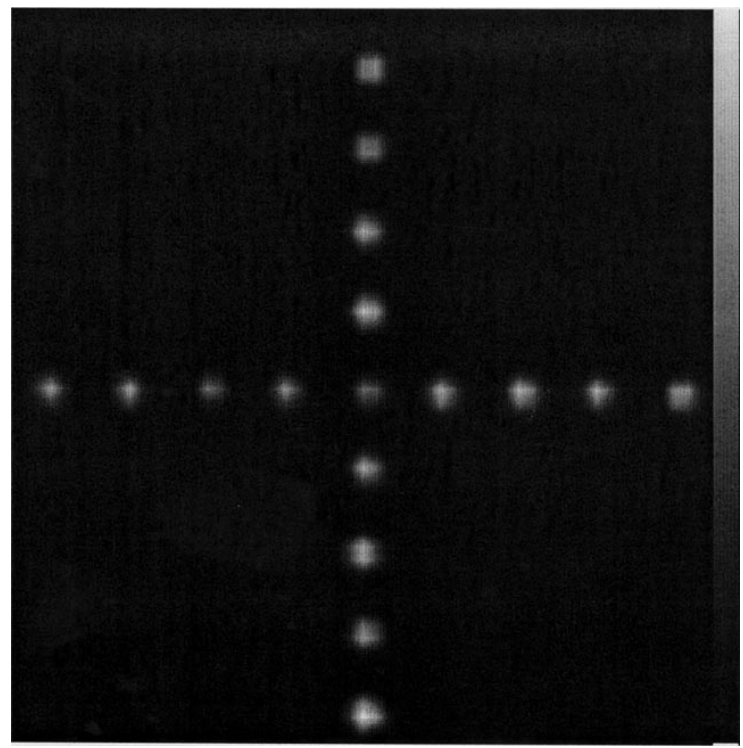

(b)

Fig. 3. (a) Photograph showing the cross pattern phantom and (b) image of the phantom acquired with the LGSO system. The dashed lines show the orientations of the horizontal and vertical profiles.

shown in this figure is a PEM image of the phantom [Fig. 3(b)]. Fig. 4(a) shows a graph of resolution calculated from horizontal profile data plotted as a function of radial distance from the center of the phantom. Fig. 4(b) shows a plot of vertical resolution versus radial position. These results reveal that there is no appreciable correlation between source position and spatial resolution. There are, however, differences in the mean resolution amongst the scintillators. The best resolution (smallest FWHM) was achieved with the LGSO-based PEM imager and the worst resolution was obtained with the $\mathrm{NaI}(\mathrm{Tl})$-based system.

The Compton scatter fraction for the three scintillators were $\mathrm{LGSO}=9 \%$, GSO $=9 \%$ and $\mathrm{NaI}(\mathrm{Tl})=8 \%$. The plots in Fig. 5 show the system energy spectra. Also shown on these plots are the fits of the photopeaks to Gaussian 

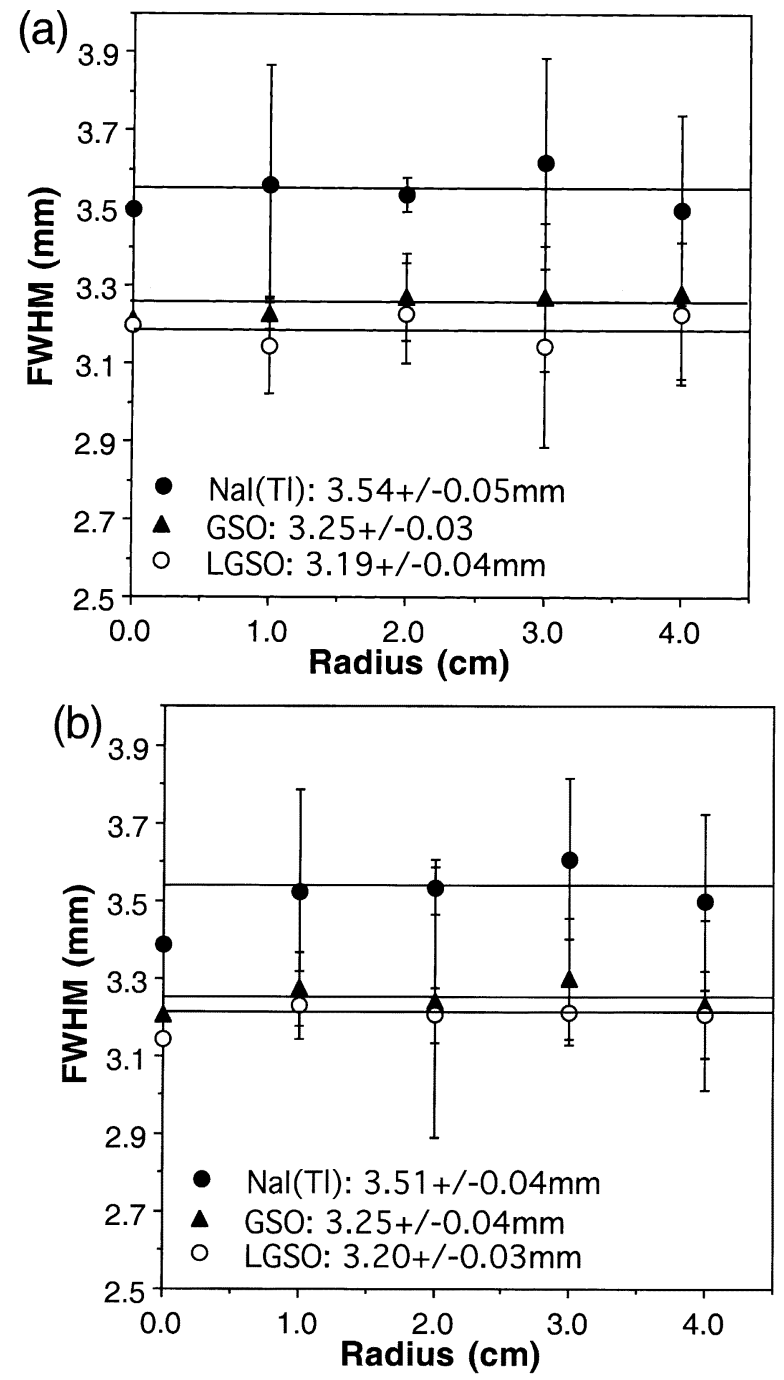

Fig. 4. Plot of resolution versus radial position of the point sources from the center of the imager for (a) horizontal and (b) vertical profiles.

functions and the resulting energy resolutions. The detection sensitivities for the three scintillators were calculated to be LGSO $=646.9 \mathrm{c} / \mathrm{s} / \mathrm{kBq} / \mathrm{ml}(23935.8 \mathrm{c} / \mathrm{s} / \mu \mathrm{Ci} / \mathrm{ml})$, $\mathrm{GSO}=383.9 \mathrm{c} / \mathrm{s} / \mathrm{kBq} / \mathrm{ml}(14203.1 \mathrm{c} / \mathrm{s} / \mu \mathrm{Ci} / \mathrm{ml})$ and $\mathrm{NaI}(\mathrm{Tl})$ $=217.7 \mathrm{c} / \mathrm{s} / \mathrm{kBq} / \mathrm{ml}(8034.4 \mathrm{c} / \mathrm{s} / \mu \mathrm{Ci} / \mathrm{ml})$. Fig. 6 shows images of the simulated breast phantom acquired with each of the three scintillators. Fig. 7 displays a plot of CNR versus sphere diameter. Note the dependence of CNR on lesion size and scintillator type.

\section{DISCUSSION}

Positron emission mammography (PEM) is a relatively new imaging technique that may become an optimal method for imaging of some women difficult to examine with standard $\mathrm{X}$-ray mammography. While a number of PEM imagers have been constructed or proposed, the identification of optimal PEM scintillators has not been systematically studied. We evaluated three candidate scintillators (LGSO, GSO, and $\mathrm{NaI}(\mathrm{Tl})$ ) for use with PEM imagers. Each of the three materials possesses potential advantages and disadvantages when applied
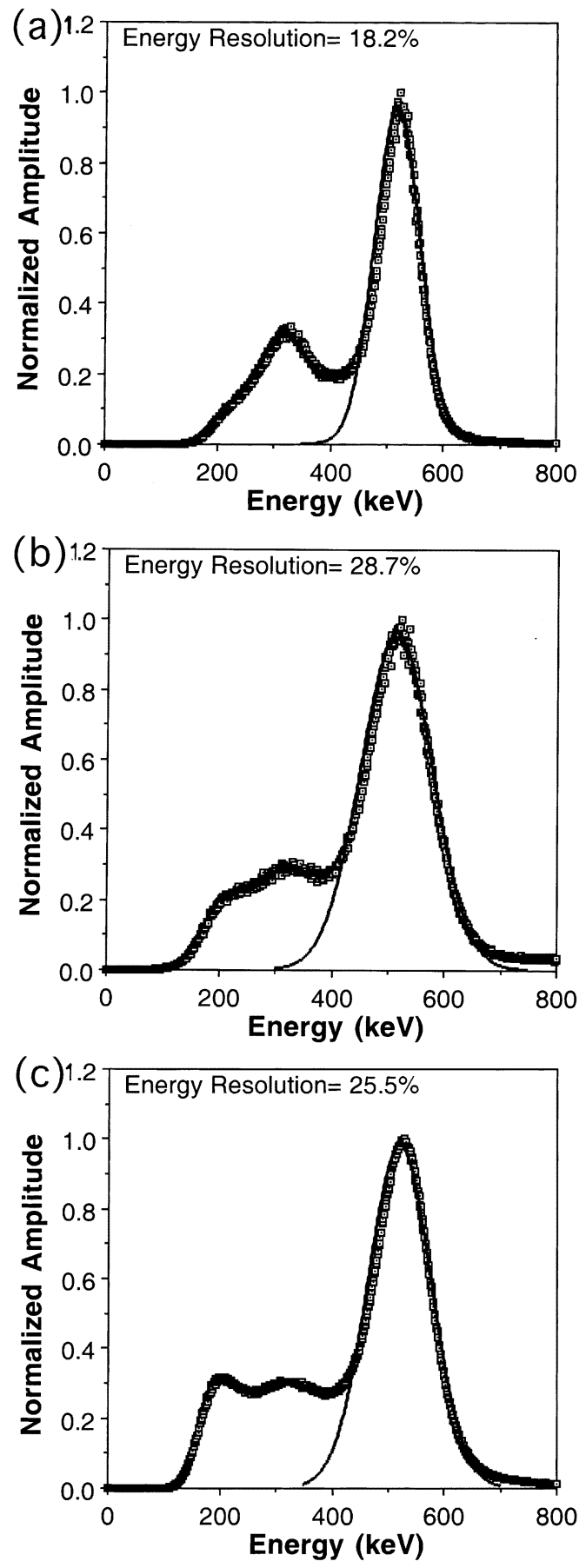

Fig. 5. System energy spectra for (a) $\mathrm{NaI}(\mathrm{Tl})$, (b) GSO, and (c) LGSO. Also shown on each plot is the calculated system energy resolution.

to PEM imaging. For example, $\mathrm{NaI}(\mathrm{Tl})$ is a low cost, high light output scintillator that is commonly used in a number of nuclear medicine applications. Unfortunately, it has relatively low stopping power for annihilation photons. The scintillator GSO is attractive because it has higher stopping power than $\mathrm{NaI}(\mathrm{Tl})$, but it has lower light output and is more expensive than $\mathrm{NaI}(\mathrm{Tl})$. Finally, LGSO has higher stopping power and produces more scintillation light than GSO, but it contains natural radioactivity and is more expensive than GSO.

Spatial resolution is particularly important for PEM imagers because it is desirable to detect relatively small breast lesions 


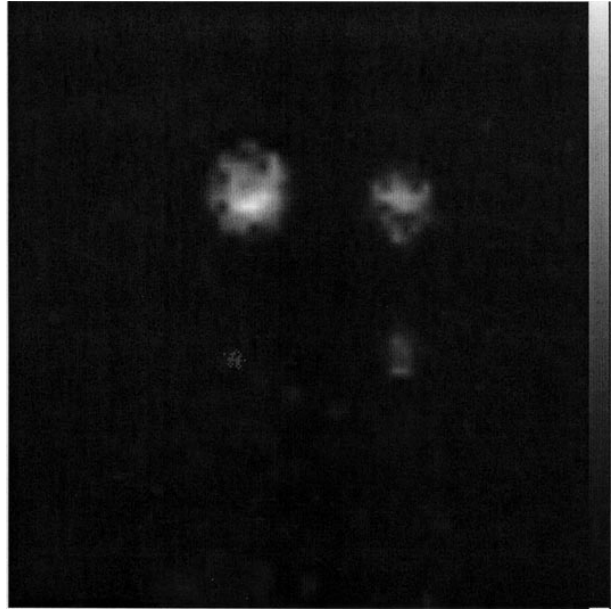

(a)

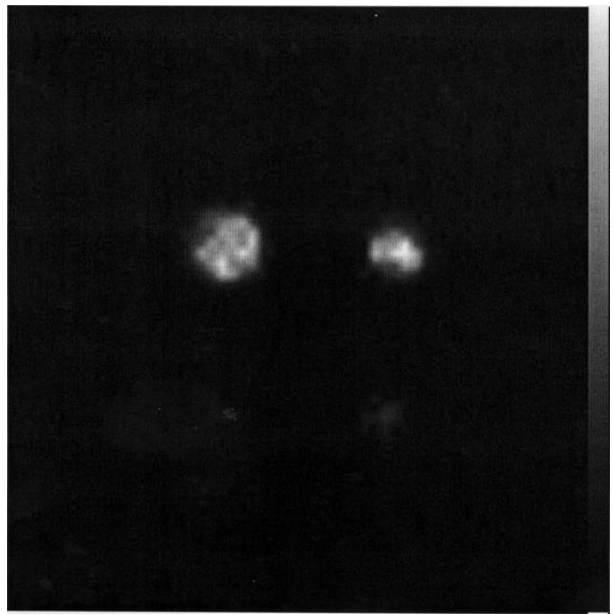

(b)

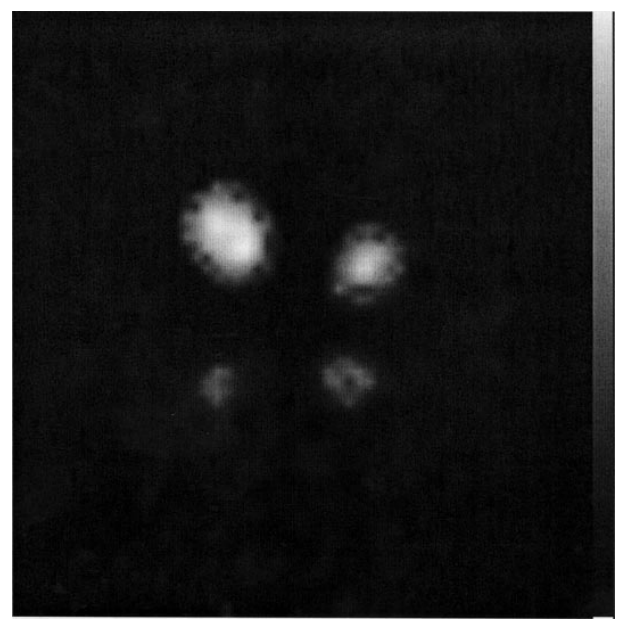

(c)

Fig. 6. PEM images of the simulated breast phantom: (a) NaI(Tl), (b) GSO and (c) LGSO.

$(<10 \mathrm{~mm}$ diameter). Although all of the PEM imagers had the same detector pixel cross-sections $\left(3 \times 3 \mathrm{~mm}^{2}\right)$, their measured spatial resolutions were not the same. Spatial resolution was found to have a dependence upon the choice of scintillator material. The $\mathrm{NaI}(\mathrm{Tl})$-based PEM system produced the poorest

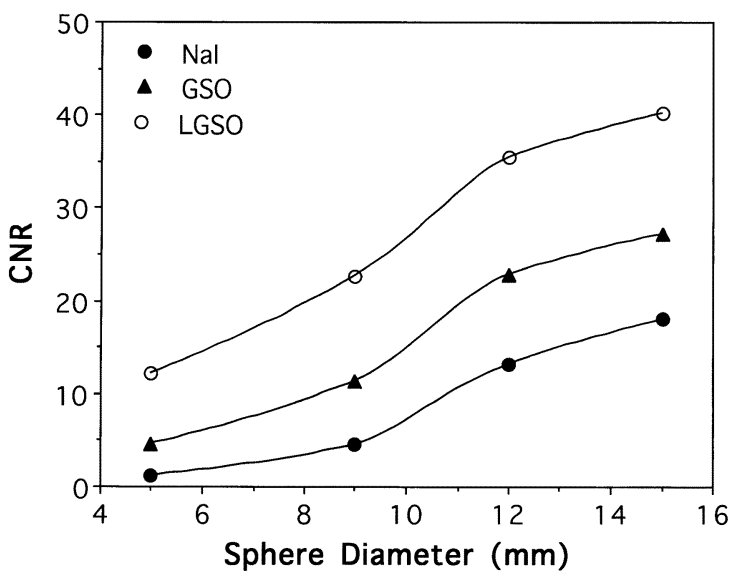

Fig. 7. Plot of CNR (4) versus sphere size.

resolution. The reduced performance of the $\mathrm{NaI}(\mathrm{Tl})$ array is likely due to factors related the physical properties of the scintillators and detector pixel thickness. First, the $\mathrm{NaI}(\mathrm{Tl})$ pixels were almost twice as long as the GSO and LGSO pixels. Thus, the scintillation light pulses became broader at the face of the PSPMTs; increasing the uncertainty in the calculation of the event centroid. This effect was reduced somewhat by the use of pixelated scintillators, which constrained the extent of broadening. Additionally, Vaquero, et al. [16], have described a phenomenon that adversely affects the measured spatial resolution of pixelated detectors. Specifically, a photon can deposit only a fraction of its energy in a detector pixel due to a Compton interaction. The scattered photon then exits the pixel, enters an adjacent pixel(s) and deposits its remaining energy. Therefore, the scintillation light can be spread over at least two pixels. The positioning algorithm will place this event along a line between the two detector pixels, broadening the PSF. These events will not be rejected on the basis of energy because the total energy deposited in the detector array (not energy per pixel) was used for discrimination. Since this effect is produced by photons exiting the primary detector pixel, the magnitude of the effect is related to the attenuation properties of the scintillator. Hence, PSF broadening should be related to the probability that a Compton scatter will occur in a pixel (assessed by the photofraction) and the likelihood that the scattered photon will exit the pixel (assessed by the linear attenuation coefficient). The large differences between the mean PSF FWHMs measured for the $\mathrm{NaI}(\mathrm{Tl})$ and GSO systems and the $\mathrm{NaI}(\mathrm{Tl})$ and LGSO systems supports this hypothesis; given the significant differences in linear attenuation coefficients and photofractions for $\mathrm{NaI}(\mathrm{Tl})$ and the other scintillators. The difference in the mean resolutions between the GSO and LGSO-based systems is relatively small, due to the small differences in linear attenuation coefficient and photofraction between the two materials (Table I). These results demonstrate that, for pixelated detector systems, the limit on spatial resolution is determined not only by the physical dimensions of the pixels but by the characteristics of the scintillator.

In addition to good spatial resolution, PEM imagers should also have good resolution uniformity. In other words, the resolution of the system should have minimal spatial dependence across the face of the detector to maximize lesion detectability 
over the whole breast. The plots in Fig. 4 demonstrate that there is relatively little correlation between imager resolution and position of a point source for all of the tested scintillators. These results are especially significant since the $\mathrm{NaI}(\mathrm{Tl})$ detector pixels were thick $(19 \mathrm{~mm})$ compared to the GSO and LGSO pixels $(10 \mathrm{~mm})$; making them more susceptible to resolution inhomogeneities caused by the parallax effect. The reason for the weak coupling of spatial resolution and source position is likely due the planar detector geometry, detector size and separation. The maximum event acceptance angle was a relatively shallow $35.3^{\circ}$ (refer to the calculation of this angle in the Methods section). Therefore, the likelihood that a photon's path would encompass multiple crystals was small.

Acceptance of Compton scattered events by the three imagers was small $(8 \%$ to $9 \%)$. These results were due to the relatively good system energy resolutions (Fig. 5) and the use of a tight energy threshold window centered upon the photopeaks. The $\mathrm{NaI}(\mathrm{Tl})$-based PEM system achieved the greatest energy resolution (18.2\%), while the GSO system produced the lowest energy resolution $(28.7 \%)$. Due to the nature of the compressed breast (a relatively thin homogeneous structure), image degradation due to the presence of Compton-scattered events should be minimal. Therefore, good energy resolution necessary to effectively reject Compton events will likely be a secondary consideration when choosing optimal detector materials for PEM imagers.

Sensitivity is an important performance metric for any detection system, but sensitivity has extra importance in PEM imaging. Since the breast is moderately compressed during PEM imaging, it is imperative to shorten imaging times to reduce discomfort and minimize the likelihood of patient motion. Thus, rapid acquisition of high-count rate image data is important for obtaining optimal PEM images. The LGSO-based system produced the greatest detection sensitivity, while the $\mathrm{NaI}(\mathrm{Tl})$-based PEM system yielded the lowest. The detection sensitivities of the PEM systems were related to the probability of photon interaction with the scintillator (measured by the total linear attenuation coefficient) and the percentage of those events that were of sufficient energy to place the events within the photopeak (gauged by the photofraction). Since the LGSO and GSO detectors were of the same thickness, much of the difference in detection sensitivity between these two materials was due to differences in attenuation coefficients and photofraction (Table I). Although the $\mathrm{NaI}(\mathrm{Tl})$ detectors were almost twice as thick as the LSGO and GSO detectors, the detection sensitivity was smaller by a factor of 1.76 compared to GSO and 2.97 compared to LGSO. Despite the fact that the probability of photon interactions for $\mathrm{NaI}(\mathrm{Tl})$ and $\mathrm{GSO}$ were similar (the product of $\mu$ and pixel thickness were nearly the same magnitude), the number of these events whose energies met the energy discrimination requirement was greatest for GSO due to its larger photofraction (Table I). The difference in detection sensitivity between GSO and LGSO was due to the larger product of $\mu$ and pixel thickness and the greater photofraction of LGSO.

The relatively poor detection sensitivity exhibited by the $\mathrm{NaI}(\mathrm{Tl})$-based system could be improved somewhat by extending the energy threshold window into the Compton plateau region of the energy spectra; thus increasing the number of accepted events. Pulse pile-up due to the increased count rate should not be a significant problem given the good performance of the pulse clipping and shaping circuitry. The increased detection sensitivity would come at the cost of increased acceptance of Compton scatter, however. This tradeoff may be acceptable, since it is likely that scatter from the breast will not be a significant source of image degradation. Another option would be to further increase the thickness of the $\mathrm{NaI}(\mathrm{Tl})$ pixels. This scheme would increase the effects of energy sharing and multicrystal penetration. Thus, unless a depth of interaction correction were instituted, the spatial resolution and resolution uniformity of the $\mathrm{NaI}(\mathrm{Tl})$-based PEM system could be significantly degraded.

Perhaps the ultimate test of a PEM system is the ability to detect radiotracer-avid breast lesions. The images shown in Fig. 6 demonstrate that the larger $(>9 \mathrm{~mm}$ diameter) spheres were well detected by all of the PEM systems. And, while the smaller spheres were detectable in all images, detection of these simulated lesions became much more challenging, especially for the $\mathrm{NaI}(\mathrm{Tl})$-based system and to a certain extent for the GSO-based system. The plots in Fig. 7 quantitatively support these observations. For all systems the CNRs were dependent upon sphere size; the smallest sphere had the lowest CNR and the largest sphere the highest. This behavior is due mostly to the fact that as the sphere size is decreased relative to the spatial resolution of the system the number of counts recovered from the image of the sphere decreases (the partial volume effect). The major cause of the stratification of the CNRs for the three systems is linked to the differences in detection sensitivity amongst the systems. Specifically, images with more detected events have more statistically uniform background regions (standard deviations in these regions are smaller compared to lower count density images). The CNRs calculated for the images produced by the higher sensitivity systems are increased; indicating improved lesion detectability. It should be noted that changing the size of the detector pixels (both the cross sectional and depth dimensions) will affect per pixel and global sensitivities, which would change lesion detectability.

Choice of scintillator material is a fundamental design consideration for virtually all radiation detection systems. The results from this study demonstrate that, for PEM imagers utilizing pixelated detector arrays, the choice of scintillator is of particular importance. Specifically, the scintillator choice significantly affects the ability of the system to detect small breast lesions. While the primary bases for selection of a scintillator for PEM systems are often the parameters measured in this investigation, other practical factors such as cost, quality and availability may also be considered. For example, while it is likely that the detection characteristics of $\mathrm{NaI}(\mathrm{Tl})$ make it nonoptimal for use with PEM systems, its low cost, good quality and good availability make in an attractive alternative for other types of pixelated imaging systems. Indeed, thick $\mathrm{NaI}(\mathrm{Tl})$ arrays with depth of interaction correction schemes may provide the basis for affordable small FOV cardiac imagers utilizing single photon emitters. Although GSO did not surpass LGSO in performance, it has practical advantages compared to LGSO. GSO is less expensive and available with more 
uniform quality than LGSO. In addition, the lack of intrinsic radiation in GSO makes it an attractive scintillator choice for PEM imagers because it reduces the need for high-speed data acquisition systems to handle the larger singles rates. It should be noted that changing the detector separation would likely change the magnitude of many of the parameters measured in this study (spatial resolution, resolution uniformity, scatter fraction, detection sensitivity). The relative differences in these parameters amongst three systems, however, should not change appreciably. In conclusion, the results from this investigation demonstrate that the utilization of pixelated detector is an important factor that can affect selection of an optimal PEM scintillator. Multicrystal scattering necessitates the use of materials with high attenuation coefficients (such as GSO and LGSO) to maintain good spatial resolution, detection sensitivity and, ultimately, good breast lesion detection capabilities.

\section{REFERENCES}

[1] C. J. Thompson, K. Murthy, I. N. Weinberg, and F. Mako, "Feasibility of positron emission mammography," Med. Phys., vol. 21, pp. 529-538, 1994.

[2] C. J. Thompson, K. Murthy, Y. Picard, and I. N. Weinberg, "Positron emission mammography (PEM): A promising technique for detecting breast cancer," IEEE Trans. Nucl. Sci., vol. 42, pp. 1012-1017, Aug. 1995.

[3] N. K. Doshi, Y. Shao, R. W. Silverman, and S. R. Cherry, "Design and evaluation of an LSO PET detector for breast cancer imaging," Med. Phys., vol. 27, pp. 1535-1543, 2000.

[4] W. W. Moses, T. F. Budinger, R. H. Huesman, and S. E. Derenzo, "PET camera designs for imaging breast cancer and axillary node involvement," J. Nucl. Med., vol. 36, p. 69P, 1995.
[5] C. L. Melcher and J. S. Schweitzer, "Cerium-doped lutetium oxy-orthosilicate: A fast, efficient, new scintillator," IEEE Trans. Nucl. Sci., vol. 39, pp. 502-505, June 1992.

[6] M. Balcerzyk, M. Moszynski, M. Kapusta, D. Wolsi, J. Pawelke, and C. L. Melcher, "YSO, LSO, and LGSO: A study of energy resolution and nonproportionality," IEEE Trans. Nucl. Sci., vol. 47, pp. 1319-1323, Aug. 2000.

[7] R. R. Raylman, S. Majewski, R. Wojcik, A. G. Weisenberger, B. Kross, and H. A. Bishop, "The potential role of positron emission mammography for detection of breast cancer. A phantom study," Med. Phys., vol. 27, pp. 1943-1954, 2000.

[8] R. Freifelder and J. S. Karp, "Dedicated PET scanners for breast imaging," Phys. Med. Biol., vol. 42, pp. 2463-2480, 1997.

[9] K. Lange and R. Carson, "EM reconstruction algorithms for emission and transmission tomography," J. Comput. Assist. Tomogr., vol. 8, pp. 306-316, 1984.

[10] "Performance Measurements of Positron Emission Tomographs," National Electrical Manufactures Association, Washington, DC, NEMA Standards Publ. NU2-1994, 1994.

[11] J. S. Karp, G. Muehllehner, D. A. Mankoff, C. E. Ordonez, J. M. Ollinger, M. E. Daube-Witherspoon, A. T. Haigh, and D. J. Beerbohm, "Continuous-slice PENN-PET: A positron tomograph with volume imaging capability," J. Nucl. Med., vol. 31, no. 5, pp. 617-27, 1990.

[12] N. Avril et al., "Breast imaging with fluorine-18-FDG PET: Quantitative image analysis," J. Nucl. Med., vol. 38, 1997.

[13] Y. Sugawara, M. A. Helvie, K. R. Zasadny, A. W. Neuhoff, S. M. Carlson, P. A. Saran, and R. L. Wahl, "Normal breast FDG uptake on PET versus body weight, age and mammographic density," J. Nucl. Med., vol. 40, pp. 248P-248P, 1999.

[14] K. R. Zasadny and R. L. Wahl, "Standardized uptake values of normal tissues at PET with 2-[fluorine-18]-fluoro-2-deoxy-D-glucose: Variations with body weight and a correction method," Radiol., vol. 189, pp. 847-850, 1993.

[15] T. G. Turkington, N. E. Williams, S. M. Hamblen, and R. E. Coleman, "Regional FDG uptake, attenuation and geometries measurements for whole body phantom design," J. Nucl. Med., vol. 40, pp. 281P-281P, 1999.

[16] J. J. Vaquero, J. Seidel, S. Siegel, W. R. Gandler, and M. V. Green, "Performance of a compact position-sensitive LSO detector module," IEEE Trans. Med. Imaging, vol. 17, pp. 967-978, Dec. 1998. 